\title{
Integration and Application of Animation Production Simplification in a VR Virtual Reality Teaching System
}

\author{
https://doi.org/10.3991/ijet.v11i10.6267 \\ Yuanyuan Ji, Shengfang Zhang \\ Dalian Jiaotong University, Dalian, Liaoning, China
}

\begin{abstract}
This study designed a teaching system based on the animation model, which focuses on VR virtual reality technology and incorporates the improved computer animation modeling technique. The whole system is composed of three main parts, including the front-end animation model, the rear-end background model and the dynamic audio model. In particular, the use of the simplified animation technology makes it easy to operate the system. On this basis, this study followed the idea of comparing experimental teaching to test the results of applying this system in the experimental class and the control class in the course of $3 \mathrm{D}$ animation production. The results confirmed that the system has a positive effect in enhancing students' interest in learning, practical ability, maturity of space cognition and so on.
\end{abstract}

Index Terms-animation production, simplification, 3D animation, VR virtual technology

\section{INTRODUCTION}

Animation is not the art form existing naturally, but has been developed due to the interaction between three seemingly unrelated fields of technology and art with the gradual development of human society, namely, art, persistence of vision and photography [1]. In the early development stage, animation was basically experimental animation. Every technological innovation would bring a qualitative leap in the animation industry [2]. In recent years, as a consequence of the continuous growth and innovation of computer software technology and hardware technology, 3D animation came into being. With the support from computer hardware, three-dimensional animation software in the computer could be used to make a fully virtual three-dimensional world. Three-dimensional animation, also known as 3D animation, is a new technology emerging with the development of computer hardware and software technology [3]. 3D animation software first establishes a virtual world in the computer. The designer builds the model and scene in the virtual threedimensional world in accordance with the shape and size of the object to be shown, sets the movement orbit of the model, movements of the virtual camera and other animation parameters according to the requirements, and finally equips the model with specific material as required, and marks with lights. When all these steps are done, the automatic operation of the computer can generate the final picture [4]. Yang used three-dimensional computer graphic, imaging technology and the motion capture system to achieve high quality and efficient three-dimensional ani- mation production. He introduced an interactive animation virtual environment construction plan based on the combination of animation, virtual reality VR equipment and network technology, and achieved desired teaching effect [5]. Ye employed virtual reality technology to produce a virtual motorcycle simulation courseware system, discussed the progress from two-dimensional design to threedimensional modeling technology and optimized technology for shaping three-dimensional scenes, the process of animation settings, and a method of using Auto CAD 3ds max Virtools to implement the technology, and finally analyzed the role of virtual reality technology-based courseware in the teaching process [6]. Merchant et al. examined the influence on the learner characteristics (such as psychological and perceptual variables) from a 3D desktop virtual reality environment which could reinforce chemistry-related learning results in an introductory class of chemistry in college. Eventually, they found an effective role of $3 \mathrm{D}$ virtual reality- based instruction in improving chemistry achievements among students [7]. Codd et al. utilized three-dimensional virtual reality technology to build a virtual human anatomy teaching system. Consequently, through using the open source 3D imaging program "Blender" in the system, they produced an interactive, three-dimensional computer model of forearm anterior compartment musculoskeletal anatomy. The objective of the following experiment was to compare the results of using traditional anatomy teaching methods and $3 \mathrm{D}$ virtual reality. Finally, as shown by the experimental results, the system is highly interactive and could help medical students improve their understanding of the human anatomy course [8]. In short, the combination of three-dimensional animation and VR technology is increasingly being applied to modern education.

However, throughout the existing research results, although 3D animation virtual technology often appears in teaching, the production of $3 \mathrm{D}$ animation requires the use of $3 \mathrm{D}$ animation production tools. Currently, it is quite difficult and complex to use 3D animation production tools. Particularly, for teachers of other majors other than animation, it is really hard to make apply $3 \mathrm{D}$ animation virtual technology according to their teaching content [9]. Consequently, they common use the PPT production system in teaching. This defect has undoubtedly hindered the promotion and development of three-dimensional virtual technology; secondly, the present teaching methods advocate student-teacher interaction. Especially in trainingoriented teaching, students engage in independent production in accordance with the topic given by the teacher. However, due to the difficulty of employing three- 
dimensional virtual technology, students suffer from some technical difficulties in completing the design work alone; besides, in the course of " $3 \mathrm{D}$ animation production" among institution of higher education, it is hard to see successful cases of the real introduction of VR technology to build the teaching system. Furthermore, relevant researches are still in a blank. Therefore, this study developed and designed a three-dimensional virtual technology production tool which can also be used by nonprofessional technical personnel (including ordinary teachers and students), and applied it in the course of "3D animation production", expecting to provide reference for the future research.

\section{DESIGN AND ANALYSIS OF THE VR ANIMATION TEACHING SYSTEM}

\section{A. Functional Analysis of the System}

To effectively solve the above-mentioned problem in face of the course of "3D animation production", it is necessary to adopt more advanced, scientific methods to design a more demonstrative, interactive and audiovisual information teaching platform, and introduce it into the teaching practice to improve the quality of curriculum teaching. Based on this design requirement, this study focused on VR virtual reality technology, incorporated the technique of animation model, and built a new threedimensional interactive dynamic teaching system. This system has many advantages in teaching, can effectively overcome various defects of the course of "3D animation production" such as many knowledge points, poor interactivity, and insufficient visual effects, and could play an extremely important role in promoting optimization of the effectiveness of teaching. Figure 1 shows the functional design of the system.

Function A: display of the animation model. Display and output of the animation model should become the primary function of the VR virtual reality technologybased system. To be specific, this function of the system includes two sub-functions: display of the front-end animation model and display of the rear-end background animation model. The function of displaying the front-end animation model is mainly used for live shows. The animation model generated can be timely presented to the audience in the 2D or 3D form for viewing and interaction; by contrast, the function of displaying the back-end animation model is employed in early production of the $2 \mathrm{D}$ or $3 \mathrm{D}$ animation to be displayed. This production is completely based on the self-adaptive process. The system can automatically set the color, model, resolution and other indicators of the animation to be displayed according to the requirement of VR display, and give the final output of the most optimal animation model.

Function B: output of the dynamic audio. Another function of this system is reflected in output of the dynamic audio. It is called output of the dynamic audio because, one the one hand, the source of the output audio can be constituted by many aspects, for example, external recording devices, including tape recorders, voice recorders, microphones and so on. During teaching, teachers can select the appropriate recording equipment according to the actual situation, to complete recording and output of the teaching audio. On the other hand, playing the audio signal can realize multi-channel output and parallel transmission. When the system is working, a set of dynamic

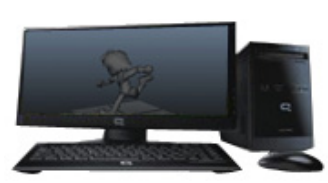

Animation model display

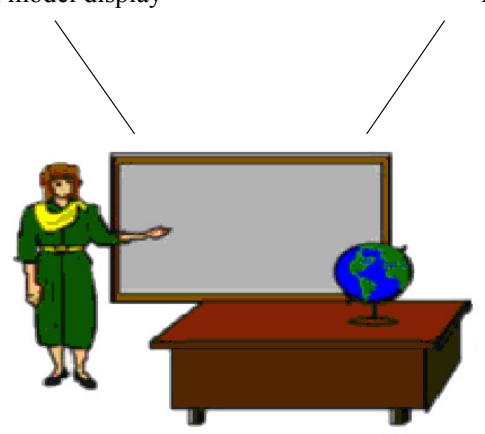

VR animation model teaching system

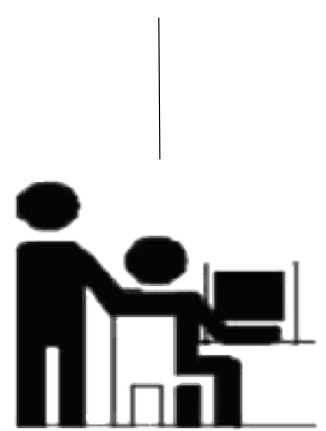

Field interactive sharing

Figure 1. Functional framework of the system

audio models can control playing of individual teaching audio. However, when teachers need to play more pieces of audio in the process of presenting the animation, they can also select the setting button for multiple sets of dynamic audio model in the system to control playing of different pieces of teaching audio simultaneously.

Function C: On-site interactive sharing. The system has a man-machine interface, using the touch-screen control technology. In practical application, users can select the 3D animation model they want to view, modify or produce through the touch control mode and engage in corresponding operations. In addition, the internal system integrates the wireless module, and comes with the LAN chatting software. With the assistance of the software, teachers and students can take part in distance learning in different spaces. Finally, the system uses SQL database technology, to build a database platform which is capable of storing data generated in the process of teaching and provide uploading, downloading, sharing, and other functions. The use of these functions enables teachers and students to share teaching experiences or results in a timely manner.

\section{B. Modules of the system}

According to the functions above, the system mainly consists of the following modules: the module of animation model display, the module of dynamic audio output, and the module of on-site interactive sharing. Figure 2 illustrates the specific modules of the system. 


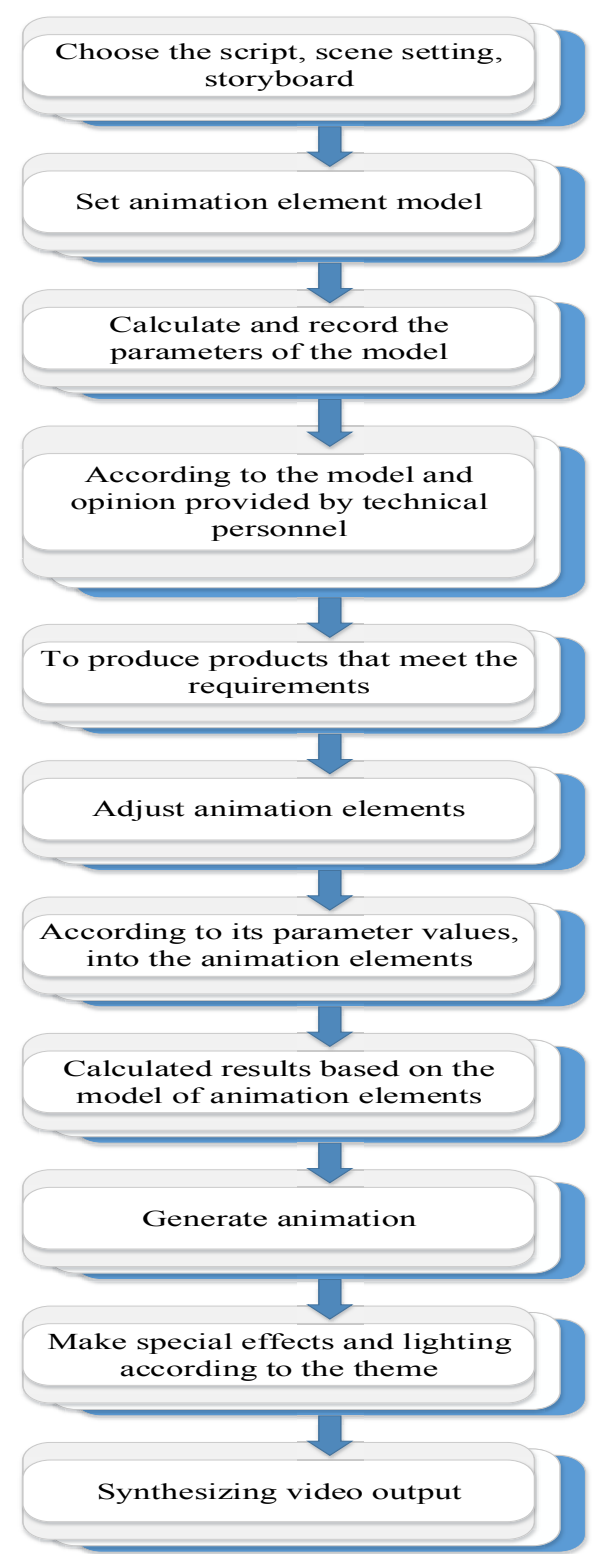

Figure 2. Diagram of overall structural frame of the system hardware

The following key technologies are applied in each module:

(1) Technologies for the module of animation model display: VRML modeling and VR-JAVA synchronizing of VR virtual reality technology are the two key technologies used in this module. Noticeably, VRML modeling technology is mainly applied for processing, rendering, and output display of the front-end and rear-end animation model in the system. Meanwhile, VR-JAVA synchronizing technology is primarily adopted for adjusting consistency of output of the front-end and rear-end animation model in the system, so as to ensure stability of the frame value of the animation model processed by the back-end and the one displayed by the front-end.

(2) Technologies for the module of dynamic audio output: parallel signal processing technique and audio output are the two technologies applied in this module. Parallel signal processing technique is mainly used for the operation of the system, to simultaneously output several sets of teaching audio signals which do not interfere with each other. Through the use of this technique, teachers can divide the objects into several groups in the teaching process, and assign different teaching task for each group, and send different audio contents of teaching demonstration by combining with the task of teaching, to achieve one-to-more and non-interfering simultaneous teaching. Audio output technology is the main technology to ensure audio signal acquisition and output in the system.

(3) Technologies for the module of on-site interactive sharing: This module mainly utilizes SQL Server database technology and touch-screen control technology. Firstly, SQL Server database technology is employed to incorporate uploading, downloading, and sharing of teaching materials, on-site exchange and other functions into the system, to achieve on-site teaching interaction. Secondly, the use of touch-screen control technology allows teachers and students to achieve instant control over display of the teaching content via this simple, direct control mode of touching.

\section{Process of using the system}

The process of using the system in teaching is shown in Figure 3. Before the class in practical applications, teachers should set up parameters related to the threedimensional animation teaching content according to the actual teaching content, for example, display parameters, audio parameters, storage parameters, etc. During teaching, the class will be divided into several groups in accordance with the learning or teaching situation, each group assigned to learn different three-dimensional animation content. Display, interpretation, sharing, storage and other functions coming with the system can be applied to demonstrate lifelike VR animation models and scenes to students and achieve interactive communications with students on-site, allowing students to experience the charm of VR virtual reality technology and thereby arousing their enthusiasm for learning.

\section{APPLICATION AND ANALYSIS OF THE VR ANIMATION TEACHING SYSTEM IN EDUCATION}

\section{A. Implementation of the teaching design}

In order to test the effect of applying the system in teaching of "3D animation production", the study specially selected Class 1 (50 students) and Class 2 (50 students) of animation enrolled in 2013 in Dalian Jiaotong University, China (located in Dalian City, Liaoning Province, China) in comparative analysis. In the specific teaching design, Class 1 was treated as the control class, and taught under the "Theory + Practice" teaching mode. It should be noted that theoretical teaching was based on the traditional PPT teaching presentation equipment. Class 2 was viewed as the experimental class and exposed to the new teaching model designed by relying on the VR animation teaching system. The specific teaching process is as follows:

\section{1) Stimulating the interest}

The teacher used the VR animation model system to give live demonstration of three-dimensional animation teaching materials for students, and stimulated students' interest in learning through powerful visual effects brought by the system. In this process, once the interest of students was inspired, the teacher should duly integrate theories and practical skills of 3D animation production, to lay the foundation for on-field practice in the later stage. 


\section{2) Getting into the scene}

Under guidance in the previous stage, the teacher took the whole class into the VR teaching training room, allowing students to experience the charm of virtual reality technology-based scenes by themselves. Meanwhile, the teacher began to guide students to make three-dimensional animation teaching materials, for example, producing key frames, building animation models, creating animation scripts, etc. On the basis of activating students' interest, the teacher led students to experience the immersive teaching environment and obtained teaching practice effects, as shown in Figure 4.

\section{3) Group teaching}

After a period of teaching, students in the class encountered different learning problems. Through LAN interactive software of the VR system, the teacher realized timely collection of students' learning problems, divided the class into several groups according to the type of their learning problems, arranged a big teaching demonstration screen for each group, and engaged in on-site exchange and explanation with students of each group by using the remote interactive feature of the system.

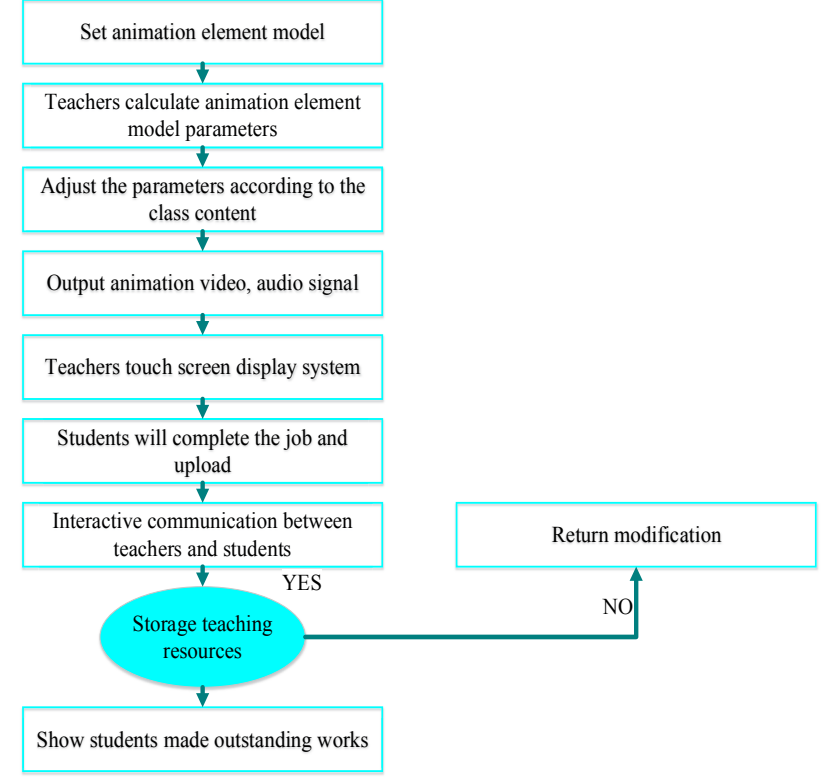

Figure 3. Process of using the system

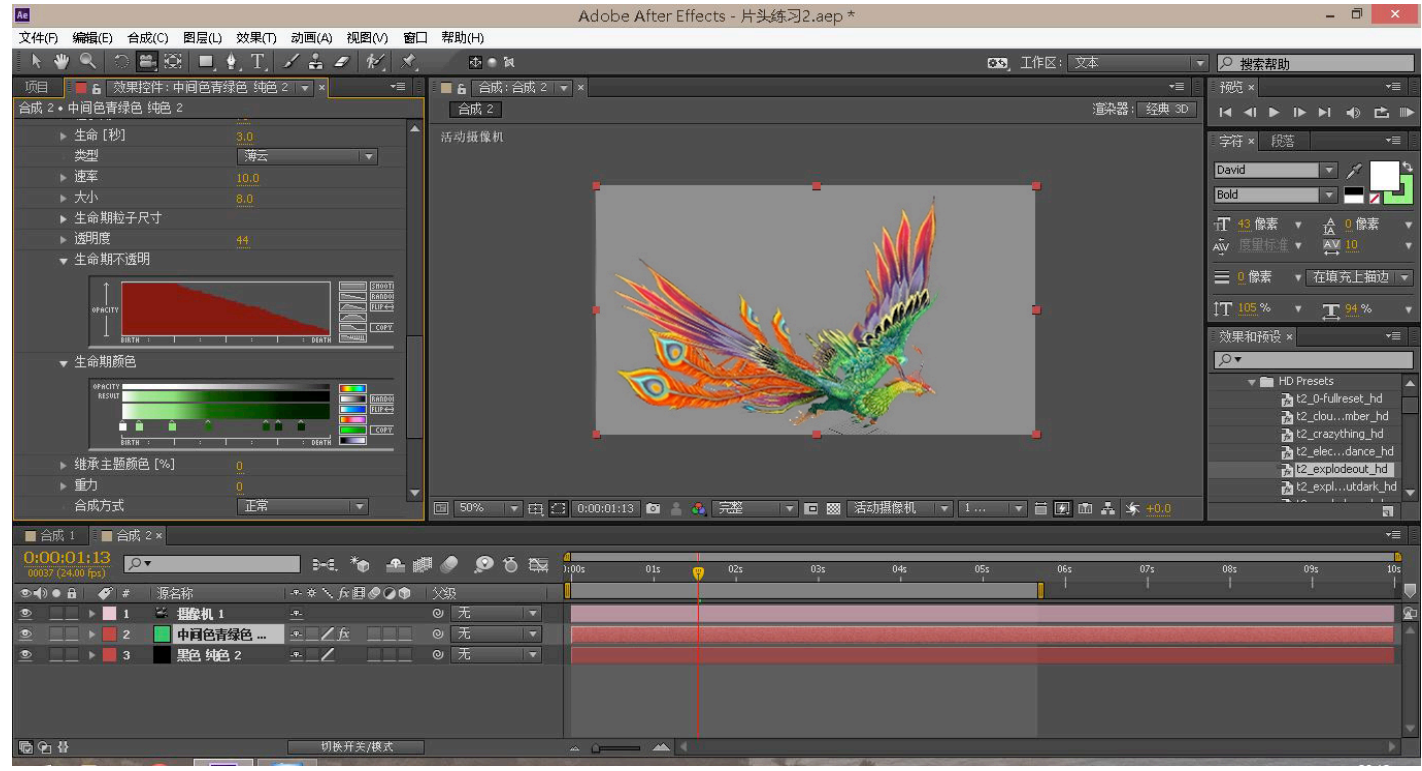

Figure 4. Process of producing three-dimensional animation

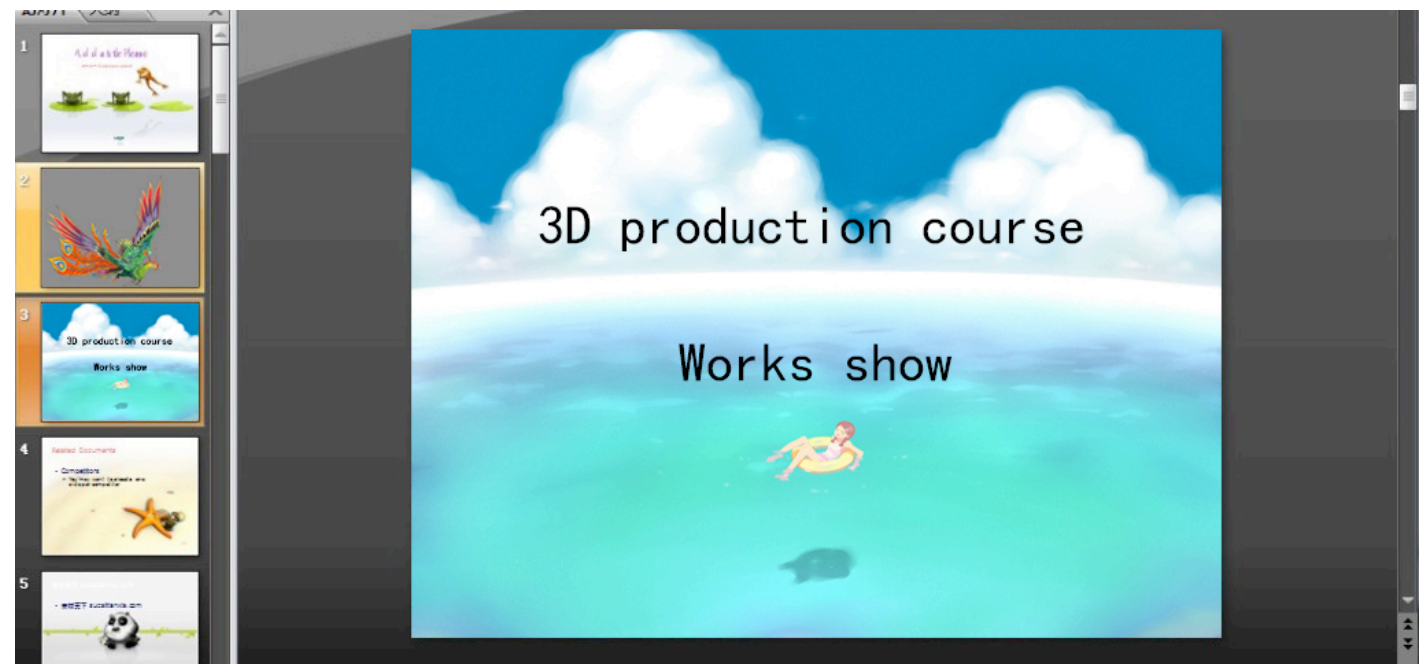

Figure 5. Group teaching for three-dimensional animation 
TABLE I.

EVALUATION OF LEARNING OUTCOMES FROM THE VR SYSTEM BY THE 50 STUDENTS IN THE EXPERIMENTAL CLASS

\begin{tabular}{lccc}
\hline \multicolumn{1}{c}{ Learning outcomes } & $\begin{array}{c}\text { Yes } \\
\text { (NO.) }\end{array}$ & $\begin{array}{c}\text { General } \\
\text { (NO.) }\end{array}$ & $\begin{array}{c}\text { No } \\
\text { (NO.) }\end{array}$ \\
\hline Enhancing learning interest & 40 & 10 & 0 \\
Enhancing practical ability & 35 & 12 & 3 \\
Enhancing spatial cognition & 44 & 6 & 0 \\
$\begin{array}{l}\text { Enhancing consolidation and review of } \\
\text { knowledge }\end{array}$ & 30 & 16 & 4 \\
\hline
\end{tabular}

\section{4) On-field practice}

After successfully answering problems of all the groups, the teacher designed hierarchical practical projects according to learning situation of each group, and led each group to use the VR animation model system in on-site production practice. When students encountered specific problems, they could exchange with the teacher onsite to find answers through the system. Finally, students uploaded the works finished to the platform via the system, for the teacher to give on-site evaluation.

\section{B. Results of Teaching Practice}

As demonstrated in the statistical teaching results in Table 1, all learning indexes of the experimental class were significantly higher than those of the control class. At the same time, analysis by the SPSS software revealed that in terms of analytical skills, curiosity, cognitive maturity and three-dimensional imagination, the control class and the experimental class did not differ in early teaching. But at the end of teaching, these figures of the experimental class were significantly better than those of the control class, which shows that the introduction of the VR animation model system indeed play a tangible role in enhancing teaching quality of "3D animation production".

In the later exchange with the experimental class, $80 \%$ of the students confirmed the advantages of the system in teaching. They mentioned that the introduction of this system makes it possible to create a more realistic and dynamic teaching scene. Compared to the conventional mode of teaching, this system is more attractive, can stimulate their interest in active learning, and could guide them to conduct in-depth study. It shows that the development and application of the teaching system is successful as a whole. However, there are some deficiencies in this teaching experiment. For example, only two classes were selected as the samples, implying that accuracy of the statistical results still should be improved. In future research and application, it is necessary to further optimize this issue.

\section{CONCLUSION}

This study designed and applied a VR animation model system. The comparative teaching study shows that: (1) its excellent audio-visual effects could greatly stimulate stu- dents' interest; (2) it has some advantages, like sound interaction, simple animation production tools and steps which are easy to master; at the same time, rich interactive classroom activities between students and teachers contribute greatly to enhancement of the teaching effectiveness of "3D animation production". Therefore, institutions of higher education are suggested to apply the teaching system into teaching of other courses of animations.

\section{REFERENCES}

[1] Yung, H.I., \& Paas, F., "Effects of cueing by a pedagogical agent in an instructional animation: a cognitive load approach," Educational Technology \& Society, vol. 18, no. 3, pp. 153-160. July 2015.

[2] Özmen, H., "Effect of animation enhanced conceptual change texts on 6th grade students' understanding of the particulate nature of matter and transformation during phase changes," Computers \& Education, vol. 57, no. 1, pp. 1114-1126, August 2011. http://dx.doi.org/10.1016/j.compedu.2010.12.004

[3] Wang, T., \& An, W.G., "The Application research of web3dbased virtual reality technology in modern distance education," Applied Mechanics and Materials, vol. 66, pp. 2216-2219, July 2011. http://dx.doi.org/10.4028/www.scientific.net/AMM.66$\underline{68.2216}$

[4] Passig, D., "Revisiting the Flynn Effect through 3D Immersive Virtual Reality (IVR)," Computers \& Education, vol. 88, pp. 327 342, October 2015. http://dx.doi.org/10.1016/j.compedu.2015.05 .008

[5] Yang, S., "Research on the combination of 3D animation and virtual reality," Microcomputer $\square$ Its Applications, vol. 30, no. 15, pp. 78-80, April 2011.

[6] Ye, H. \& Zhang, R.J., "Experiment Based on Virtual Reality Technology Production and Application of Courseware," Journal of Anshun College, vol. 12, no. 1, pp. 91-93, September 2010.

[7] Merchant, Z., Goetz, E.T., Keeney-Kennicutt, W., Kwoka, O, Cifuentesa, L., Davisc, T.J., "The learner characteristics, features of desktop 3D virtual reality environments, and college chemistry instruction: A structural equation modeling analysis," Computers \& Education, vol. 59, no. 2, pp. 551-568, September 2012. http://dx.doi.org/10.1016/j.compedu.2012.02.004

[8] Codd, A.M., Choudhury, B., "Virtual reality anatomy: Is it comparable with traditional methods in the teaching of human forearm musculoskeletal anatomy?" Anatomical Sciences Education, vol. 4, no. 3, pp. 119-125, April 2011. http://dx.doi.org/10.1002/ase. 214

[9] Mikropoulos, T.A., "Presence: a unique characteristic in educational virtual environments," Virtual Reality, vol. 10, no. 3-4, pp. 197-206, September 2006. http://dx.doi.org/10.1007/s10055-0060039-1

\section{AUTHORS}

Yuanyuan Ji is a Lecturer of Dalian Jiaotong University, Dalian 116028, Liaoning, China. Her research interests include 3D Technology and Digital Animation Production. (zhangshengfang1973@163.com)

Shengfang Zhang (Corresponding author) is a lecture in Dalian Jiaotong University, Dalian 116028, Liaoning, China. Her research interests include virtual classroom and distance education (zhangshengfang1973@163.com)

Submitted 23 September 2016. Published as resubmitted by the authors 24 October 2016. 